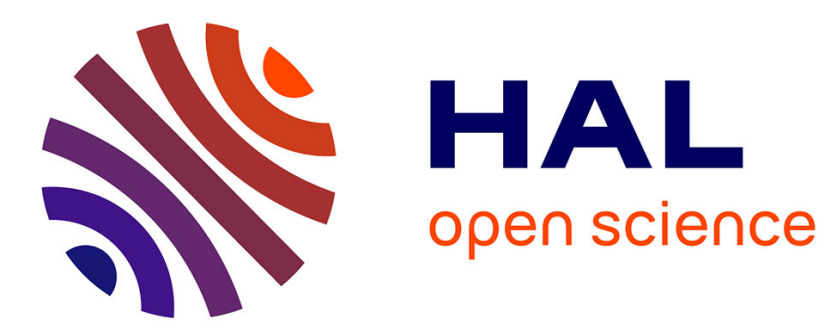

\title{
Brief Announcement: Distributed Exclusive and Perpetual Tree Searching
}

Lélia Blin, Janna Burman, Nicolas Nisse

\section{To cite this version:}

Lélia Blin, Janna Burman, Nicolas Nisse. Brief Announcement: Distributed Exclusive and Perpetual Tree Searching. DISC 2012 - 26th International Symposium on Distributed Computing, Oct 2012, Salvador, Brazil. pp.403-404, 10.1007/978-3-642-33651-5_29 . hal-00741982

\section{HAL Id: hal-00741982 \\ https://hal.inria.fr/hal-00741982}

Submitted on 15 Oct 2012

HAL is a multi-disciplinary open access archive for the deposit and dissemination of scientific research documents, whether they are published or not. The documents may come from teaching and research institutions in France or abroad, or from public or private research centers.
L'archive ouverte pluridisciplinaire HAL, est destinée au dépôt et à la diffusion de documents scientifiques de niveau recherche, publiés ou non, émanant des établissements d'enseignement et de recherche français ou étrangers, des laboratoires publics ou privés. 


\title{
Brief Announcement: Distributed Exclusive and Perpetual Tree Searching*
}

\author{
Lélia Blin ${ }^{1 \star \star}$, Janna Burman ${ }^{2}$, and Nicolas Nisse ${ }^{3 \star \star \star}$ \\ 1 Univ. d'Evry Val d'Essone, et LIP6, Univ. Pierre et Marie Curie, Paris, France \\ ${ }^{2}$ Grand Large, INRIA, LRI, Orsay, France \\ 3 MASCOTTE, INRIA, I3S(CNRS/UNS), Sophia Antipolis, France
}

\begin{abstract}
We tackle a version of the well known graph searching problem where a team of robots aims at capturing an intruder in a graph. The robots and the intruder move between the graph nodes. The intruder is invisible, arbitrary fast, and omniscient. It is caught whenever it stands on a node occupied by a robot, and cannot escape to a neighboring node. We study graph searching in the CORDA model of mobile computing: robots are asynchronous and perform cycles of Look-Compute-Move actions. Moreover, motivated by physical constraints and similarly to some previous works, we assume the exclusivity property, stating that no two or more robots can occupy the same node at the same time. In addition, we assume that the network and the robots are anonymous. Finally, robots are oblivious, i.e., each robot performs its move actions based only on its current "vision" of the positions of the other robots. Our objective is to characterize, for a graph $G$, a set of integers such that for every integer $k$ in the set, perpetual graph searching can be achieved by a team of $k$ robots starting from any $k$ distinct nodes in $G$. One of our main results is a full characterization of this set, for any asymmetric tree. Towards providing a characterization for all trees, including trees with non-trivial automorphisms, we have also provided a set of positive and negative results, including a full characterization for any line. All our positive results are based on the design of graph searching algorithms.
\end{abstract}

\section{Introduction}

This BA announces a work that aims at understanding the algorithmic power and limitation of computing with autonomous mobile robots. The literature dealing with this objective has considered different kinds of coordination tasks, including pattern formation, robot gathering, and graph exploration. Each of these tasks involves complex coordination protocols for the robots, whose complexity depends on the capabilities of the robots in terms of perception of their environment, individual computational power, and communication. The CORDA model has been introduced for capturing the essence of mobile computing. It focusses on the asynchronous nature of the actions performed by the robots, and on the limitations caused by the absence of communication between them.

\footnotetext{
* Based on: L. Blin, J. Burman, and N. Nisse: Perpetual Graph Searching, Research Report INRIA-RR-7897, Sophia-Antipolis, France, Feb. 2012.

** Additional support from the ANR project "SHAMAN".

$\star \star \star$ Partially supported by FP7 STREP EULER (N.N.)
} 
In this model, robots are endowed with visibility sensors allowing each robot to perceive the positions of all the other robots. They operate in asynchronous Look-Compute-Move action cycles. During its look action, a robot perceives the relative positions of the other robots; during the compute action, it executes some individual deterministic computation whose input is the set of the latest perceived positions of the other robots (which may have changed since they were measured); finally, during the move action, the robot changes its position according to its computation.

The coordination of autonomous deterministic mobile robots has been first studied in continuous environments (e.g., the 2-dimensional Euclidean space). In the discrete CORDA model, it is not clear whether even simple coordination tasks can be achieved. As a consequence, most of the literature on robot computing assume additional hypotheses, including presence of identities enabling to distinguish robots, capacity to store the sequence of all previous perceived positions and moves, sense of direction, and the ability to construct towers of robots (i.e., to bring several robots at a node). We have proved that a very complex task, like graph searching, can be solved without any of these assumptions. We assume that the robots have no identities, they are memoryless (i.e., stateless), have no sense of direction, and two or more robots cannot occupy the same position at the same time (exclusivity property). We call min-CORDA this essential CORDA model.

\section{Contributions}

For any graph $G$, let $\mathrm{fs}(G)$ denote the set of robot team sizes such that $k \in \mathrm{fs}(G)$ if and only if distributed graph searching in $G$ can be achieved by a team of $k$ robots starting from any $k$ (distinct) nodes. Our main result consists in a characterization of $\mathrm{fs}(T)$, for any asymmetric tree $T$. Let $\mathrm{xs}(T)$ denote the exclusive search number, i.e., the minimum number of robots to be used for capturing the intruder in a centralized setting satisfying the exclusivity property. By definition, $k \in \mathrm{fs}(T)$ implies that $k \geq \mathrm{xs}(T)$. We have proved that, when $T$ possesses no symmetries (i.e., has no non-trivial automorphisms), then $k \in \mathrm{fs}(T)$ for all $k \geq \mathrm{xs}(T)+1$. This result is based on the explicit design of a distributed protocol enabling perpetual graph searching by $k$ robots, for any $k \geq \mathrm{xs}(T)+1$.

When $T$ possesses symmetries, the computation of $\mathrm{fs}(T)$ becomes more complex. In this case, we have shown that $\mathrm{fs}(T)$ depends on the set $\mathcal{S}_{T}$ of isomorphic nodes of $T$ separated by a path of even length. In particular, $k \notin \mathrm{fs}(T)$ for any $k \in\left[\left|\mathcal{S}_{T}\right|,\left|\mathcal{S}_{T}\right|+\mathrm{xs}\left(T_{0}\right)\right]$, where $T_{0}$ results from $T$ after removing all nodes appearing in $\mathcal{S}_{T}$. Our impossibility results are not based on the perpetual nature of graph searching in min-CORDA, but on the exclusivity property. For the simpler case of a line, we have fully characterized $\mathrm{fs}(L)$ for any $n$-node line $L$. All our positive results are constructive.

We note that exclusive graph searching behaves very different from the classical one. As a result and due to min-CORDA, the proofs and the algorithms we propose are very different and more involved than in the classical case. Our proofs introduce several techniques that may prove useful also in future studies of exclusive graph-searching and min-CORDA. 